\title{
A MOTIVACIÓN DO ALUMNADO E O VALOR ATRIBUÍDO AOS INSTRUMENTOS DE AVALIACIÓN
}

\author{
Rodríguez-Llorente, Carolina'; Piñeiro Aguín, Isabel'; \\ González-Suárez, Rocío3 ; Martins Ginorelli, Ludmila ${ }^{4}$ \\ ${ }^{1}$ Universidade da Coruña, Facultade de Ciencias da Educación, \\ https://orcid.org/0000-0002-2894-5271 \\ ${ }^{2}$ Universidade da Coruña, Facultade de Ciencias da Educación, \\ https://orcid.org/0000-0002-1845-2238 \\ ${ }^{3}$ Universidade da Coruña, Facultade de Ciencias da Educación, \\ https://orcid.org/0000-0002-7065-1015 \\ ${ }^{4}$ Universidade da Coruña, Facultade de Ciencias da Educación, \\ https://orcid.org/0000-0002-9527-4295
}

\section{RESUMO}

A situación derivada da pandemia provocada pola COVID-19 transformou substancialmente as metodoloxías de ensino-aprendizaxe. Unha mudanza moi evidente deuse nos instrumentos de avaliación ao teren que se adaptar ás modalidades de ensino online e semipresencial. 0 grupo de innovación GIPED-INNOVA deseñou unha investigación có obxectivo de coñecer os instrumentos de avaliación que se empregaron durante 0 curso académico, o valor que lle dan os estudantes e como este varía en función da motivación académica destes. No estudo participaron 178 estudantes das materias que imparten os docentes de GIPED-INNOVA. Para a recollida da información, elaborouse un cuestionario online ad hoc no que se incluíron os distintos instrumentos que se poden empregar para avaliar 0 rendemento do alumnado na Universidade da Coruña. Por outra banda, para coñecer a motivación dos estudantes utilizouse o Cuestionario de Metas Académicas. Segundo os resultados, os participantes consideran que os docentes de GIPED-INNOVA empregan en maior medida instrumentos de avaliación relativos ás prácticas de aula. Ademais, os estudantes que adoptan metas de aprendizaxe valoran 
especialmente estas ferramentas xunto a asistencia a clase. Non obstante, nin os estudantes con metas de rendemento nin aqueles con metas de aprendizaxe valoran os instrumento tipo proba.

PALABRAS CLAVE: motivación académica, instrumentos de avaliación, universidade, psicoloxía educativa.

\section{CITA RECOMENDADA:}

Rodríguez Llorente, Carolina; Piñeiro Aguín, Isabel; González Suárez, Rocío; Martins Ginorelli, Ludmila (2021): A motivación do alumnado e o valor atribuído aos instrumentos de avaliación. En García Naya, J.A. (ed.) (2021). Contextos universitarios transformadores: a nova normalidade académica. Leccións aprendidas e retos de futuro. V Xornadas de Innovación Docente. Cufie. Universidade da Coruña. A Coruña (pág. 325-337).

DOI capítulo: https://doi.org/10.17979/spudc.9788497498180.325

DOI libro: https://doi.org/10.17979/spudc. 9788497498180

\section{ABSTRACT}

The COVID-19 pandemic has significantly altered the teaching-learning methodologies. The evaluation instruments used to measure students' performance constituted one of the most noticeable changes on this new online teaching context. The group of teaching innovation GIPED-INNOVA designed a research that aims to shed light on the evaluation instruments used during the current academic year, the value with which the students regarded them and how it varies regarding student's academic motivation. $A$ hundred and seventy-eight students participated in the study. All of them attended the GIPED-INNOVA professors' classes. An online questionnaire, which included the different evaluation instruments that can be used to measure students' performance at the University of A Coruña, was designed ad hoc. On the other hand, the Goal Orientation Scale was used to obtain information regarding students' academic motivation. According to the results, participants requested that the GIPED-INNOVA's professors mainly used tasks and class exercises as evaluation instruments. Moreover, those who adopt a mastery goal orientation valued both these instruments and class attendance the most. However, neither the students that held mastery goals nor those who adopted performance goals valued exams.

KEY WORDS: academic motivation, academic assessment tools, university, educational psychology. 


\section{INTRODUCIÓN}

As metodoloxías de ensino-aprendizaxe de corte innovador están cada vez máis presentes nas guías docentes dos títulos de grao e mestrado das universidades, seguindo as indicacións do Plan Bolonia (Fernández e Ezkurra, 2020). Concretamente, a aplicación destas innovacións na docencia ten que estar dirixida a facilitar, apoiar e/ou mellorar a aprendizaxe. Agora ben, durante os dous últimos cursos, marcados pola pandemia da COVID-19, semella que a innovación educativa perdeu forza debido ao contexto de ensino virtual e ao posterior modelo de educación semipresencial. Ao mesmo tempo, esta situación pode ter provocado unha diminución significativa da motivación do alumnado (Gil et al., 2020). Polo tanto, resulta relevante concretar que recursos están a empregar os docentes nos procesos de ensinoaprendizaxe e en que medida os estudantes universitarios considéranos valiosos.

Para dar resposta a estas cuestións, a principios do ano académico constituíuse 0 Grupo de Innovación Educativa GIPED-INNOVA, cuxo propósito fundamental consiste en promover 0 coñecemento acerca da calidade das metodoloxías docentes utilizadas no contexto da Universidade da Coruña (UDC) e a percepción que ten delas o alumnado, desde a perspectiva da Psicoloxía Educativa. Deste xeito, deseñouse un proxecto de investigación para que 0 profesorado da UDC poda coñecer a percepción que teñen os estudantes sobre as metodoloxías que empregan nas súas materias.

Un dos principais cambios na docencia durante a pandemia e na nova normalidade déronse nos métodos de avaliación, por exemplo, aumentando a presenza de instrumentos como as traballos do alumnado e as probas online (García-Peñalvo et al., 2020). Como 0 emprego de certos recursos de avaliación estaría estreitamente relacionado coa motivación dos estudantes (Campos-Mesa et al., 2019), un dos obxectivos do proxecto de investigación do GIPEDINNOVA é coñecer como está a ser avaliado o seu alumnado e se os recursos que empregan son valiosos para estes. Tamén pretende analizar se hai diferenzas no valor có que os estudantes perciben eses instrumentos segundo a súa motivación. 


\section{MARCO TEÓRICO}

0 Espazo Europeo de Educación Superior (EEES) introduzo numerosas novidades na docencia universitaria, pasando a ser 0 estudante 0 protagonista do proceso de ensino-aprendizaxe (Álvarez, 2005). Este feito, así como a aparición de conceptos como o de competencia, requiriu da adaptación dos métodos de avaliación á nova realidade educativa nas universidades.

A avaliación constitúe unha das partes fundamentais para coñecer a eficacia dos procesos de ensino-aprendizaxe en calquera dos niveis educativos. Deste xeito, Contreras (2004), define a avaliación como un proceso no que se obtén información sobre 0 dominio que 0 alumnado ten do aprendido (p. ex., conceptos, habilidades), se emiten xuízos de valor (p. ex., sobresaínte, axeitado, insuficiente) e se toman decisións (p. ex., promocionar ou non promocionar).

Neste proceso de avaliación poden empregarse unha ampla variedade de recursos ou instrumentos que teñen por finalidade recoller información relativa aos aprendizaxes do alumnado (Álvarez, 2005). Concretamente, na elaboración das guías docentes das materias dos diferentes títulos ofrecidos pola UDC, os docentes poden elixir entre 11 instrumentos de avaliación: documentos onde se recollen actividades e reflexións (portafolios); distintos tipos de probas (oral, escrita en formato test ou escrita con cuestións de desenvolver); exposición individual ou grupal; tarefas ou prácticas de aula; resolución de casos, supostos ou situacións; traballos ou proxectos; participación nas actividades de aula; puntualidade coas entregas das tarefas establecidas; e asistencia ás sesións de aula.

Esta diversidade de ferramentas para avaliar o rendemento dos estudantes contrasta coas empregadas noutras etapas educativas, nas que aínda predominan as probas escritas. Non hai dúbida de que os exames ofrecen información sobre a adquisición das aprendizaxes, mais poden provocar altos niveis de ansiedade no alumnado (Domínguez-Laro et al., 2017) e ata levalos a recorrer a prácticas deshonestas (Sureda-Negre et al., 2009). Isto resulta de especial relevancia pola situación na que se atopa o sistema universitario á hora de avaliar utilizando esta técnica, que en moitos casos adaptáronse a un formato online. Lonxe de obter 
información de calidade sobre a adquisición de coñecementos destrezas e actitudes, as probas electrónicas non deron as mesmas oportunidades a todo 0 alumnado (García-Peñalvo et al., 2020). Por iso, estase a favoreces a combinación de diferentes recursos para medir 0 nivel de logro dos mesmos obxectivos.

A avaliación é tamén relevante porque está vinculada á motivación do alumnado. Por exemplo, segundo o recurso ou recursos de avaliación empregados, os estudantes poderían percibilos ben como unha oportunidade para aprender, ben como unha simple cualificación (Álvarez, 2005). Unha das variables máis utilizadas para medir a motivación dos estudantes no contexto académico é a orientación a metas. Descrita como o conxunto de razóns dos estudantes para implicarse na súa aprendizaxe, o alumnado podería adoptar unha orientación a metas de aprendizaxe, é dicir, para mellorar as súas capacidades, ou a metas de rendemento, isto é, para demostrar a súa capacidade de conseguir boas notas en relación aos compañeiros (Elliot e Dweck, 1988). Seguindo todas estas ideas e tendo en conta que 0 alumnado ten un papel activo nos seus procesos de aprendizaxe, parece interesante coñecer cales son os recursos de avaliación que os estudantes consideran valiosos segundo os seus motivos para implicarse cos mesmos.

Polo tanto, os obxectivos deste estudo son (a) examinar a frecuencia de uso dos diferentes instrumentos de avaliación utilizados polo grupo de docente de GIPED-INNOVA nas distintas materias que imparten e (b) analizar se 0 valor que os alumnos e alumnas lle atribúen aos instrumentos de avaliación é diferente segundo a súa orientación a metas. As hipóteses de partida suxiren que aqueles estudantes que adopten metas de aprendizaxe valorarán máis aquelas tarefas relacionadas cos traballos de clase e proxectos. Por outro lado, espérase que os estudantes con metas de rendemento perciban as probas individuais como un instrumento de avaliación máis valioso. 


\section{METODOLOXÍA}

\subsection{PARTICIPANTES}

No estudo participaron 178 estudantes (85.95\% mulleres) dos sete membros con docencia de GIPED-INNOVA. Os participantes, de idades comprendidas entre os 18 e os 45 anos $\left(\mathrm{M}_{\text {idade }}=\right.$ 21.94; SD $=4.01$ ), estaban a estudar algunha das 11 materias nas que teñen docencia os profesores do grupo de innovación, as cales están repartidas en cinco títulos de grao e dous de mestrado da Facultade de Ciencias da Educación. Por curso, no momento da recollida da información 0 21.8\% dos participantes cursaban o primeiro ano de grao, o 12.9\% o segundo ano, $038.2 \%$ o terceiro ano, o 1.1\% o cuarto ano e o 19.7\% eran alumnos e alumnas de mestrado.

\subsection{INSTRUMENTOS}

Nesta investigación deseñouse un cuestionario online en Microsoft Forms composto por diferentes escalas para recoller a información relativa á motivación académica do alumnado, a frecuencia coa que os estudantes perciben que os seus docentes empregan os distintos instrumentos de avaliación nas súas materias e o valor que estes lles asignan.

En primeiro lugar, preguntouse ao alumnado polo nome do docente e a materia en base aos cales ían contestar ás seguintes cuestións. Tamén se lles pediu que indicaran a titulación e 0 curso no que estaban a estudar.

En segundo lugar, para obter información sobre a motivación do alumnado cos seus estudos empregouse o Cuestionario de Metas Académicas (CMA) (Skaalvik, 1997). Con este cuestionario avalíase a orientación a metas de aprendizaxe (ou aproximación á tarefa) e metas de aproximación ao rendemento. Trátase dunha escala tipo Likert con cinco opcións de resposta por cada afirmación (onde 1 = nunca e $5=$ sempre) formada por 10 preguntas que permiten diferencias entre aqueles estudantes que adoptan metas de aprendizaxe (5 ítems; $\alpha$ $=.84$ ) (ítems exemplo: "É importante para min aprender cosas novas en clase"; ou "O que 
aprendo nas clases desta materia faime querer aprender máis") e metas de rendemento (5 ítems; $\alpha=.73$ ) (ítems exemplo: "Intento conseguir notas máis altas que outros estudantes nesta materia"; ou "Nesta materia, intento facelo mellor que outros estudantes").

Por último, para coñecer o uso que fan os docentes de GIPED-INNOVA dos diferentes instrumentos de avaliación e o valor co que os estudantes perciben cada un deles, deseñouse un cuestionario creado ad hoc con dúas subescalas tipo Likert, ambas formadas por unha serie de preguntas relativas a cada unha das ferramentas de avaliación que poden utilizarse no deseño das guías docentes da UDC. No caso da frecuencia de uso dos instrumentos de avaliación polo profesorado de GIPED-INNOVA, as opcións de resposta foron desde 1 ou até 5 ou moitas veces (ítems exemplo: "Proba oral", "Traballos ou proxectos" ou "Proba escrita en formato test"), mentres que para indicar o seu valor os estudantes podías responder desde 1 ou nada até 5 ou moito.

\subsection{PROCEDEMENTO}

0 proxecto de investigación deseñouse tendo presentes os principios éticos establecidos na Declaración de Helsinki e seguindo as recomendacións do Comité de Ética da Investigación e da Docencia da UDC ( $\mathrm{n}^{0}$ de expediente 2021-007). Para garantir 0 anonimato dos participantes o persoal investigador do equipo que se encargou da recollida, tratamento e análise dos datos non tiña relación co alumnado. 0 instrumento empregado para obter a información do estudo estivo dispoñible no campus virtual nas materias impartidas polos docentes de GIPED-INNOVA. Os estudantes interesados en participar na investigación responderon, polo tanto, de maneira voluntaria ás preguntas formuladas fora do horario lectivo.

\subsection{ANÁLISE DOS DATOS}

En primeiro lugar, examináronse os estatísticos de descritivos das variables estudadas. A continuación, realizouse a análise factorial exploratoria da escala creada ad hoc para coñecer 
0 valor dos instrumentos de avaliación, que deu como resultado tres factores: primeiro, un conxunto de instrumentos de avaliación relativas á Asistencia (2 ítems; $\alpha=.78$ ) (ítem exemplo: "Asistencia ás sesións de aula”); segundo, un factor que engloba Tarefas para realizar na aula (3 ítems; $\alpha=.74$ ) (ítems exemplo: "Traballos o proxectos"; ou "Resolución

de casos, supostos ou situacións"); e, finalmente, un factor Probas (3 items; $\alpha=.72$ ) (items exemplo: "Proba escrita en formato test"; ou "Proba oral"). A proba de esfericidade de Bartlett $\left(X^{2}=402.388 ; g l=28 ; p<.001\right)$ sosteñen a adecuación da análise e o KM0 (.675) informa da correcta suficiencia de mostreo.

En segundo lugar, para coñecer as diferenzas no valor dos instrumentos de avaliación percibido polos estudantes segundo a súa motivación académica, empregando como variables independentes a orientación a metas de rendemento e de aprendizaxe, leváronse a cabo dúas análises de varianza (ANOVA). Como medida do tamaño do efecto utilizouse o coeficiente eta cadrado parcial $\left(\mathrm{n}_{\mathrm{p} 2}\right)$. Para a súa interpretación tomouse 0 criterio establecido por Cohen (1998), segundo o cal un efecto é pequeno cando $\eta_{\mathrm{p} 2}=.01(d=.20)$, 0 efecto é medio cando $\eta_{\mathrm{p} 2}=.059(d=.50)$ e 0 tamaño 0 efecto é grande cando $\eta_{\mathrm{p} 2}=.138(d=.80)$.

\section{RESULTADOS}

Segundo os resultados, os estudantes que participaron na investigación informan de que os seus docentes empregan con maior frecuencia como ferramentas de avaliación as Tarefas de aula $(\mathrm{M}=4.37 ; \mathrm{SD}=.87)$ e tamén a Asistencia a clase $(\mathrm{M}=3.99$; $\mathrm{SD}=.78)$. Por outra banda, consideran que as Probas non son utilizadas na mesma medida que as anteriores para este propósito ( $\mathrm{M}=2.44 ; \mathrm{SD}=.90$ ). Na Figura 1 poden observarse os resultados sobre a percepción do alumnado respecto a frecuencia coa que os docentes empregan os diferentes tipos de instrumentos de avaliación. 


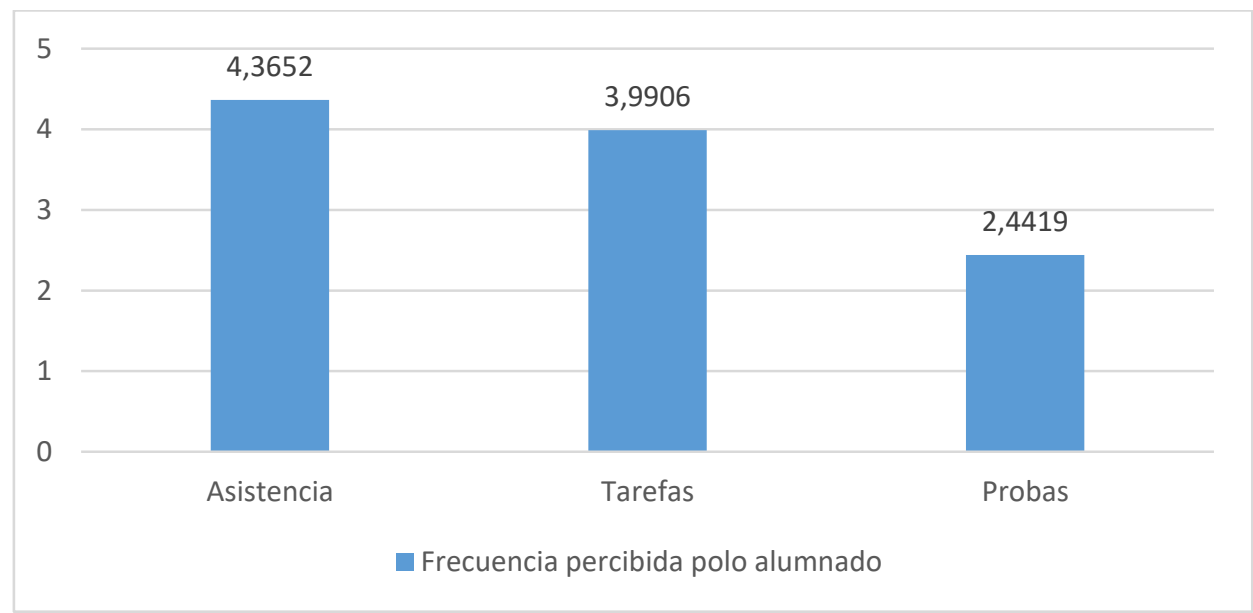

Figura 1. Frecuencia dos instrumentos de avaliación empregados polos docentes de GIPED-INNOVA percibida polo alumnado

Atendendo ao segundo obxectivo do estudo, atopouse unha correlación estatisticamente significativa de carácter positivo entre as metas de aprendizaxe e a Asistencia a clase $(r=$ .24) e as Tarefas de aula $(r=.29)$. Por outra banda, as metas de aprendizaxe non presentan unha correlación estatisticamente significativa coas Probas e, no caso das metas de rendemento, non 0 farían con ningún dos instrumentos de avaliación. Así, as alumnas e alumnos que presentan unha maior tendencia cara as metas de aprendizaxe, valorarían máis a avaliación que teña en conta a puntualidade e asistencia e tarefas como a resolución de casos ou a elaboración de proxectos (Ver Táboa 1).

\begin{tabular}{lccccc}
\hline & 1 & 2 & 3 & 4 & 5 \\
\hline 1. Metas de aprendizaxe & 1 & & & & \\
2. Metas de rendemento & .040 & 1 & & & \\
3. Asistencia & $.235^{\mathrm{a}}$ & -.035 & 1 & & \\
4. Tarefas & $.288^{\mathrm{a}}$ & -.047 & $.383^{\mathrm{a}}$ & 1 & \\
5. Probas & .025 & .073 & .004 & .061 & 1 \\
\hline
\end{tabular}

${ }^{\mathrm{a}} p<0.01$

Táboa 1. Matriz de correlación da orientación a metas e do valor dos instrumentos de avaliación 
A ANOVA levada a cabo tomando como factor a orientación a metas de aprendizaxe constatou diferenzas significativas, con tamaños do efecto pequenos, no valor da Asistencia $\left(F_{(13,428)}=\right.$ $\left.2.857 ; p<.01 ; \eta_{\mathrm{p} 2}=.16\right)$ e das Tarefas $\left(F_{(13,428)}=6.741 ; p<.001 ; \eta_{\mathrm{p} 2}=.31\right)$. Non obstante, non se atoparon diferenzas estatisticamente significativas entre os tres tipos de valoración cando se tomou como factor a orientación a metas de rendemento.

\section{CONCLUSIÓNS}

GIPED-INNOVA, desde a perspectiva da Psicoloxía Educativa, pretende analizar as prácticas docentes do profesorado que forma parte do equipo e facer extensivos os seus descubrimentos a outras titulacións. Concretamente, neste estudo analizáronse os instrumentos de avaliación que empregan nas súas materias, o valor que se asignan os estudantes a eses instrumentos e en que medida este último depende da súa motivación académica.

En primeiro lugar, semella que 0 alumnado destes docentes considera que empregan con maior frecuencia instrumentos de avaliación relativos á asistencia a clase e á realización de tarefas do que probas. Ao mesmo tempo, os estudantes tamén perciben como ferramentas más valiosas as dúas primeiras. Así, de xeito xeral, os estudantes outórganlles máis valor a recursos como a resolución de casos ou a asistencia a clase.

Por outra banda, en canto a relación entre a motivación do alumnado e 0 valor dos instrumentos de avaliación semella que, mentres que os estudantes que adoptan unha orientación a metas de rendemento non valoran ningún dos instrumentos de avaliación que empregan os seus docentes, aqueles que sosteñen metas de aprendizaxe valoran especialmente que os seus profesores teñan en conta para á avaliación a asistencia e a puntualidade, xunto con os traballos e proxectos que realizan para á materia. Finalmente, ningún dos tipos de metas se relaciona con unha valoración positiva dos exames.

A relevancia desta investigación reside, en primeiro lugar, nas súas posibles repercusións na calidade da docencia no ámbito universitario. Os resultados deste estudo permitiron aos 
membros docentes de GIPED-INNOVA mellorar 0 seu coñecemento sobre a súa práctica no que respecta á avaliación académica, partindo das percepcións que teñen os seus alumnos sobre os instrumentos de avaliación que empregan e o valor que lles outorgan. Alén diso, tamén comprobaron que, dependendo da motivación do alumnado, tomando como compoñente a orientación a metas, valoran máis uns instrumentos fronte a outros. Toda esta información é relevante para 0 deseño das guías docentes, facéndoas más adaptadas 0 interese e valoracións do alumando.

Por outro lado, enténdese que estudar a repercusión que os distintos instrumentos de avaliación teñen no proceso de aprendizaxe axudará a optimizar a docencia especialmente nas circunstancias nas que se está a desenvolver (Caskurlu et al., 2021). Por iso, GIPED-INNOVA pretende continuar a investigar nesta liña para coñecer os efectos do uso dos diferentes instrumentos de avaliación na motivación do alumnado, incorporando compoñentes como as expectativas de éxito ou a autoeficacia.

\section{REFERENCIAS}

Álvarez, M. B. (2005). Adaptación del método docente al Espacio Europeo de Educación Superior: La motivación de los alumnos como instrumento clave. Estudios sobre Educación, 9, 107-126. https://doi.org/10.15581/004.9.\%25p

Campos-Mesa, M., González-Campos, G., \& Castañeda-Vázquez, C. (2019). Análisis de la motivación del estudiante de educación superior participante en una propuesta de evaluación formativa. SPORT TK-Revista EuroAmericana de Ciencias del Deporte, 8(2), 53-58. https://doi.org/10.6018/sportk.401111

Caskurlu, S., Richardson, J. C., Alamri, H. A., Chartier, K., Farmer, T., Janakiraman, S., Strait, M., \& Yang, M. (2021). Cognitive load and online course quality: Insights from instructional designers in a higher education context. British Journal of Educational Technology, 52, 584-605. https://doi.org/10.1111/bjet.13043378 
Contreras, E. (2004). Evaluación de los aprendizajes universitarios. En R. Rodríguez, J. Hernández e S. Hernández (Coords.), Docencia universitaria. Orientaciones para la formación del profesorado (pp. 129-152). ICE. Universidad de Oviedo.

Domínguez-Lara, S. A., Alarcón-Parco, D., \& Navarro-Loli, J. S. (2017). Relación entre ansiedad ante exámenes y rendimiento en exámenes en universitarios: análisis preliminar de la diferencia según asignatura. Revista Digital de Investigación en Docencia Universitaria, 11(1), 166-176. http://dx.doi.org/10.19083/ridu.11.492

Elliot, E. S., \& Dweck, C. S. (1988). Goals: An approach to motivation and achievement. Journal of Personality and Social Psychology, 54, 5-12. https://doi.org/10.1037/00223514.54.1.5

Fernández, I. \& Ezkurra, A. M. (2020). La transformación docente de la universidad a 20 años de Bolonia: Balance y claves para un futuro por definir. Profesorado. Revista de Currículum y Formación de Profesorado, 24(1), 28-52. https://www.doi.org.10.30827/profesorado.v24i2.15149382Gil

García-Peñalvo, F. J., Abella-García, V., Corell, A., \& Grande, M. (2020). La evaluación online en la educación superior en tiempos de la COVID-19. Education in the Knowledge Society, 21(12), 1-26. https://doi.org/10.14201/eks.23013

Gil, F., Urchaga, J. D., \& Sánchez, A. (2020). Percepciones y expectativas en el alumnado universitario a partir de la adaptación a la enseñanza no presencial motivada por la pandemia de COVID-19. Revista Latina, (78), 65-85. https://doi.org/10.4185/RLCS2020-1470

Pekrun, R., Goetz, T., Frenzel, A. C., \& Perry, R. P. (2011). Achievement Emotions

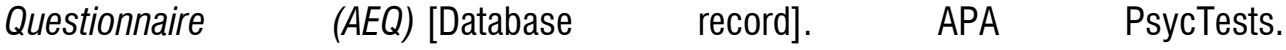
https://doi.org/10.1037/t21196-000

Pintrich, P. R., Smith, D. A., Garcia, T., \& McKeachie, W. J. (1991). A manual for the use of the Motivated Strategies for Learning Questionnaire (MSLQ). Ann Arbor, MI: National Center for Research to Improve Postsecondary Teaching and Learning. 
Skaalvik, E. (1997). Self-enhancing and self-defeating ego orientation: Relations with task and avoidance orientation, achievement, self-perceptions, and anxiety. Journal of Educational Psychology, 89(1), 71-81. https://doi.org/10.1037/0022-0663.89.1.71

Sureda-Negre, J., Comas-Forgas, R., \& Gili-Planas, M. (2009). Prácticas académicas deshonestas en el desarrollo de exámenes entre el alumnado universitario español. Estudios sobre Educación, 17, 103-122. https://doi.org/10.15581/004.17.\%25p 
